\title{
A novel endoscopic suturing device after endoscopic full-thickness resection of gastric submucosal tumor
}

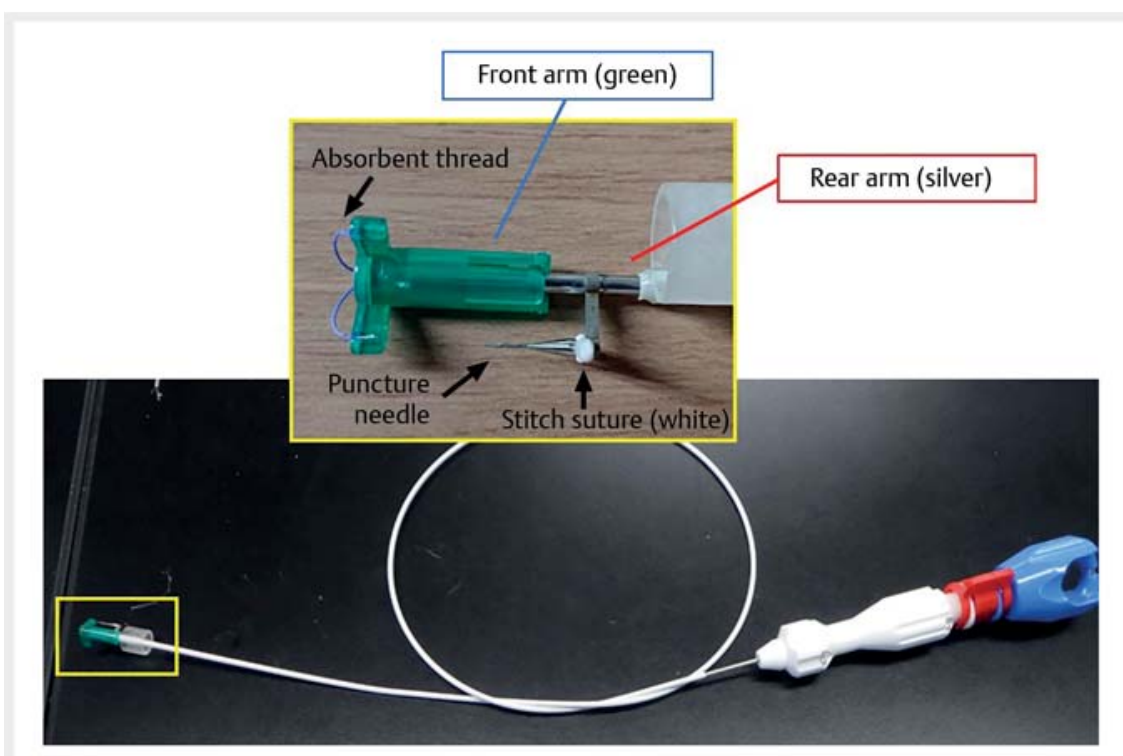

- Fig. 1 Endoscopic suturing device (Zeosuture M). This device has two arms (yellow flame). The rear arm (silver) has the puncture needle and the stitch suture (white), and the front arm (green) has the absorbent thread.

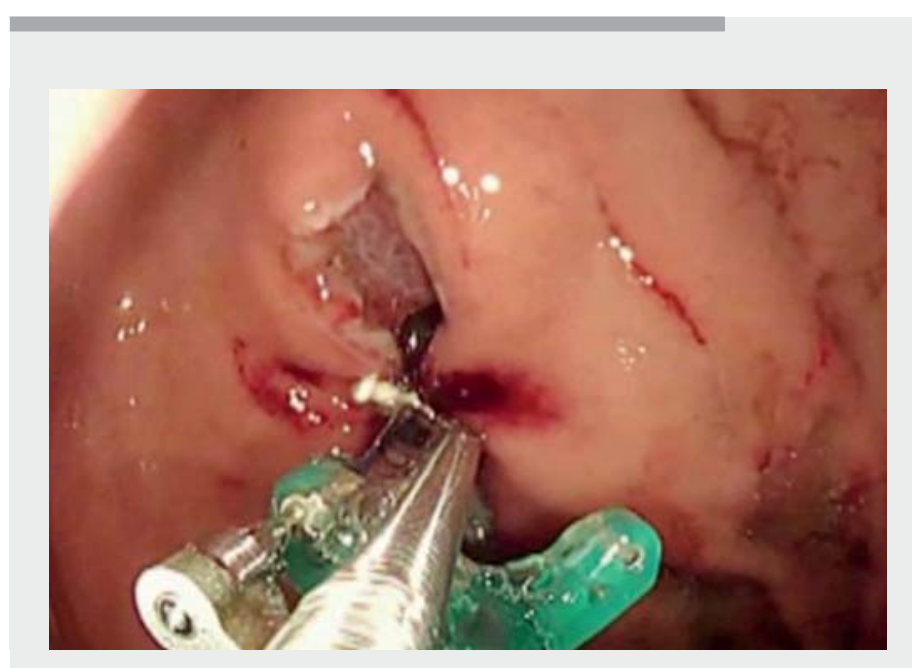

Video 1 The wall defect after endoscopic full-thickness resection of gastric gastrointestinal stromal tumor was closed by three-stitch sutures using Zeosuture M.

Endoscopic full-thickness resection (EFTR) is a minimally invasive technique that has shown promising efficacy in the resection of gastrointestinal submucosal tumors. The key to a successful EFTR procedure is the complete closure of the wall defect to prevent peritonitis and the need for surgical intervention [1,2]. Here, we present a suturing technique to close a gastric wall defect after perform-

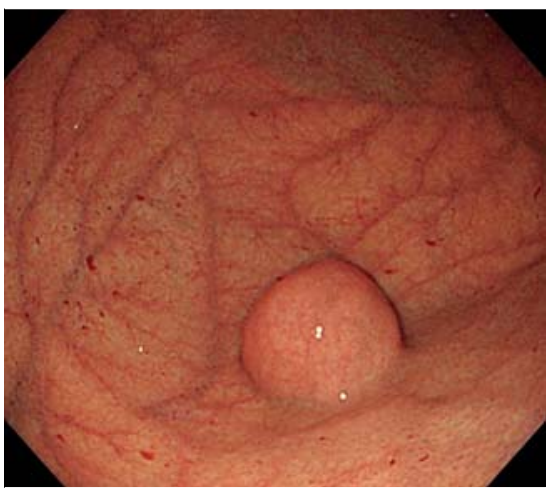

- Fig. 2 Gastric gastrointestinal stromal tumor was seen in the fornix.

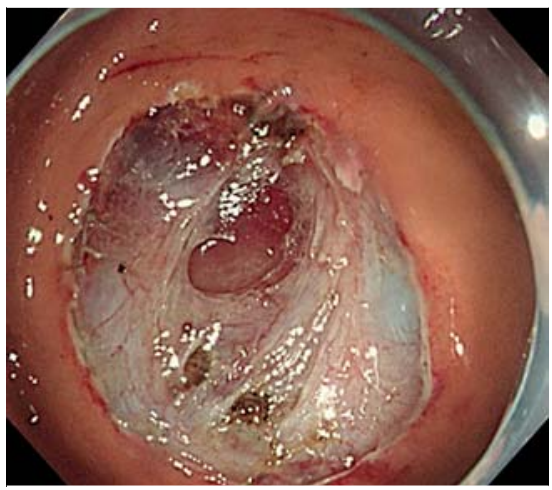

- Fig. 3 Ulcer floor after endoscopic fullthickness resection.

ing EFTR with Zeosuture M (Zeon Medical Co., Tokyo, Japan), a novel endoscopic suturing device ( $\triangleright$ Fig. 1 ).

A 46-year-old man was diagnosed with a 25-mm intra-growth gastric gastrointestinal stromal tumor (GIST) in the fornix ( $\triangleright$ Fig.2) ( $\downarrow$ Video 1). The EFTR was performed with a ring-thread counter traction ( $\triangleright$ Fig. 3). The endoscopic closure of the wall defect was performed using Zeosuture $M$ through a single-channel endoscope. First, one end of the front arm was inserted into the edge of the serosal side, and the rear arm with the puncture needle was moved forward and penetrated the full thickness of the resected margin. When the absorbent thread and the connector joined the front arm and the punc- 


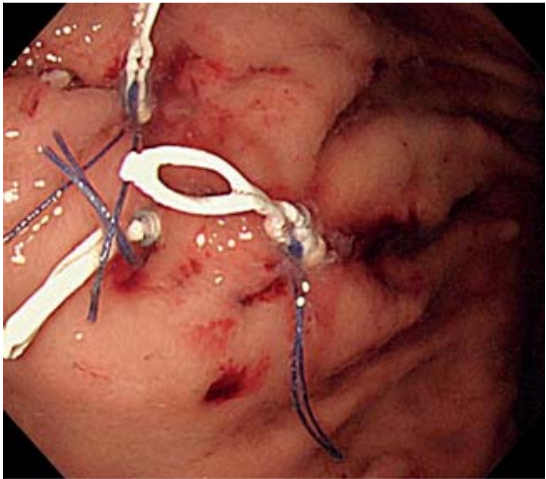

- Fig. 4 Three-stitch sutures were placed, and a complete closure was obtained.

ture needle, they were pulled out from the gastric mucosa. Then the rear arm was rotated to the opposite side. This arm was placed at the resection opening and the puncture needle was passed through the full thickness. Next, the full thickness of both resected margins of the resection opening was tied and tension was applied to the thread by Zeotieupper S (Zeon Medical). Then, ligation was performed. Finally, the thread was cut with Hookcutter MI (Zeon Medical). In a similar manner, the wall defect and post-EFTR ulcer floor were successfully closed by three-stitch sutures at an approximately 5-mm interval ( $\mathbf{F i g . 4 ) . ~}$ Follow-up endoscopy on post-operative day 14 revealed the sustained closure of the wall defect ( $\triangleright$ Fig. 5).

Hence, Zeosuture $M$ is a novel full-thickness suturing device and can be a reliable option for suturing the wall defect after EFTR.

Endoscopy_UCTN_Code_TTT_1AO_2AI

Competing interests

The authors declare that they have no conflict of interest.

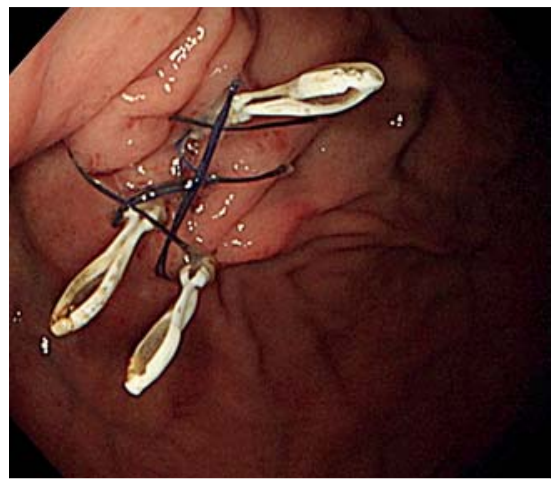

Fig. 5 Suture site on postoperative day 14 .

The authors

Hideyuki Chiba ${ }^{1}$, Ken Ohata ${ }^{2}$, Hirohito Mori $^{3,4}$, Hiroki Kuwabara ${ }^{1}$, Yoshinori Sato ${ }^{3}$, Yosuke Tsuji ${ }^{5}$, Fumio Itoh ${ }^{3}$

1 Department of Gastroenterology, Omori Red Cross Hospital, Tokyo, Japan

2 Department of Gastrointestinal Endoscopy, NTT Medical Center, Tokyo, Japan

3 Department of Internal Medicine, Division of Gastroenterology and Hepatology, St. Marianna University School of Medicine, Kawasaki, Kanagawa, Japan

4 Department of Gastroenterology, Ehime Rosai Hospital, Nihama-shi, Ehime, Japan

5 Department of Gastroenterology, Graduate School of Medicine, The University of Tokyo, Tokyo, Japan

\section{Corresponding author}

\section{Hideyuki Chiba, MD}

Department of Gastroenterology, Omori Red Cross Hospital, 4-30-1 Chuo, Ota-ku, Tokyo 143-8527, Japan h.chiba04@gmail.com

\section{References}

[1] Mori H, Kobara H, Fujihara S et al. Feasibility of pure EFTR using an innovative new endoscopic suturing device: The Double-arm-bar Suturing System (with video). Surg Endosc 2014; 28: 683-690

[2] Goto O, Oyama T, Ono H et al. Endoscopic hand-suturing is feasible, safe, and may reduce bleeding risk after gastric endoscopic submucosal dissection: a multicenter pilot study (with video). Gastrointest Endosc 2020; 91: 1195-1202

Bibliography

Endoscopy 2022; 54: E419-E420

DOI 10.1055/a-1581-7679

ISSN 0013-726X

published online 8.9.2021

(c) 2021. Thieme. All rights reserved.

Georg Thieme Verlag KG, Rüdigerstraße 14, 70469 Stuttgart, Germany

\section{ENDOSCOPY E-VIDEOS}

https://eref.thieme.de/e-videos

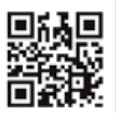

Endoscopy E-Videos is an open access online section, reporting on interesting cases and new techniques in gastroenterological endoscopy. All papers include a high quality video and all contributions are freely accessible online. Processing charges apply (currently EUR 375), discounts and wavers acc. to HINARI are available.

This section has its own submission website at https://mc.manuscriptcentral.com/e-videos 